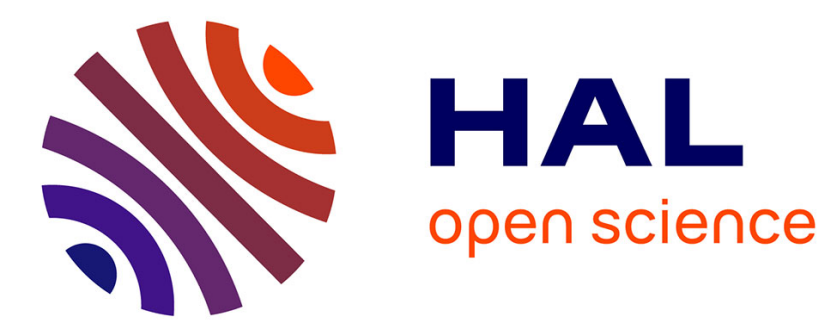

\title{
Oriented vertex and arc colorings of outerplanar graphs
}

Alexandre Pinlou, Eric Sopena

\section{To cite this version:}

Alexandre Pinlou, Eric Sopena. Oriented vertex and arc colorings of outerplanar graphs. Information Processing Letters, 2006, 100, pp.97-104. hal-00307093

\section{HAL Id: hal-00307093 \\ https://hal.science/hal-00307093}

Submitted on 3 Nov 2008

HAL is a multi-disciplinary open access archive for the deposit and dissemination of scientific research documents, whether they are published or not. The documents may come from teaching and research institutions in France or abroad, or from public or private research centers.
L'archive ouverte pluridisciplinaire HAL, est destinée au dépôt et à la diffusion de documents scientifiques de niveau recherche, publiés ou non, émanant des établissements d'enseignement et de recherche français ou étrangers, des laboratoires publics ou privés. 


\title{
Oriented vertex and arc colorings of outerplanar graphs
}

\author{
Alexandre Pinlou*, Éric Sopena ${ }^{\dagger}$ \\ LaBRI, Université Bordeaux 1, \\ 351, cours de la Libération \\ 33405 Talence Cedex, France
}

March 30, 2006

\begin{abstract}
A homomorphism from an oriented graph $G$ to an oriented graph $H$ is an arc-preserving mapping $\varphi$ from $V(G)$ to $V(H)$, that is $\varphi(x) \varphi(y)$ is an arc in $H$ whenever $x y$ is an arc in $G$. The oriented chromatic number of $G$ is the minimum order of an oriented graph $H$ such that $G$ has a homomorphism to $H$. The oriented chromatic index of $G$ is the minimum order of an oriented graph $H$ such that the line-digraph of $G$ has a homomorphism to $H$.

In this paper, we determine for every $k \geq 3$ the oriented chromatic number and the oriented chromatic index of the class of oriented outerplanar graphs with girth at least $k$.
\end{abstract}

Keywords: Outerplanar graph, girth, oriented chromatic number, oriented chromatic index.

Mathematical Subject Classification: 05C15

\section{Introduction}

In this paper, we consider oriented graphs, that is digraphs without opposite arcs. For an oriented graph $G$, we denote by $V(G)$ its set of vertices, by $A(G)$ its set of arcs, and

\footnotetext{
*Part of this work has been supported by the European training network COMBSTRU.

${ }^{\dagger}$ E-mail: $\{$ pinlou, sopena\}@labri.fr
}

by $u v$ an arc from vertex $u$ to vertex $v$. The number of vertices of $G$ is the order of $G$.

The girth of a graph $G$ is the size of a smallest cycle in $G$. We denote by $\mathcal{O}_{g}$ the class of oriented outerplanar graphs with girth at least $g$.

An oriented $k$-vertex-coloring of an oriented graph $G$ is a mapping $\varphi$ from $V(G)$ to a set of $k$ colors such that $(i) \varphi(u) \neq \varphi(v)$ whenever $u v \in A(G)$ and $(i i) \varphi(v) \neq \varphi(x)$ whenever $u v, x y \in A(G)$ and $\varphi(u)=\varphi(y)$. Note that these two conditions ensure that any two vertices linked by a directed path of length one or two are assigned distinct colors in any oriented vertex-coloring.

The oriented chromatic number of $G$, denoted by $\chi_{o}(G)$, is the smallest integer $k$ such that $G$ admits an oriented $k$-vertex-coloring. The oriented chromatic number $\chi_{o}(\mathcal{F})$ of a class of oriented graphs $\mathcal{F}$ is defined as the maximum of $\chi_{o}(G)$ taken over all graphs $G$ in $\mathcal{F}$.

Let $G$ and $H$ be two oriented graphs. A homomorphism from $G$ to $H$ is a mapping $\varphi$ from $V(G)$ to $V(H)$ that preserves the arcs (that is $\varphi(u) \varphi(v) \in A(H)$ whenever $u v \in$ $A(G)$ ). An oriented $k$-vertex-coloring of an oriented graph $G$ can thus be viewed as a homomorphism from $G$ to $H$, where $H$ is an oriented graph of order $k$. The existence of such 
a homomorphism from $G$ to $H$ is denoted by $G \rightarrow H$. The vertices of $H$ are called colors, and we say that $G$ is $H$-vertex-colorable. The oriented chromatic number of $G$ can then be equivalently defined as the smallest order of an oriented graph $H$ such that $G \rightarrow H$.

The notion of oriented vertex-coloring, introduced by Courcelle in [3], has been studied by several authors in the last decade and the problem of bounding the oriented chromatic number has been investigated for various graph classes (see $[1,2,6,8,9,11]$, or [10] for an overview).

Concerning outerplanar graphs, Sopena proved in [9] that the class of oriented partial 2-trees (which contains the class of oriented outerplanar graphs) has oriented chromatic number at most 7 and that this bound is tight (he provided an outerplanar graph with oriented chromatic number 7). In [4], Hosseini Dolama constructed an oriented trianglefree outerplanar graph with oriented chromatic number 6 and asked whether the oriented chromatic number of the class of oriented triangle-free outerplanar graphs is 6 or 7 . Our first result gives a complete classification of the oriented chromatic numbers of oriented outerplanar graphs with given girth:

\section{Theorem 1}

$$
\begin{aligned}
& \text { 1. } \chi_{o}\left(\mathcal{O}_{4}\right)=6 \\
& \text { 2. } \chi_{o}\left(\mathcal{O}_{g}\right)=5 \text { for every } g, g \geq 5 \text {. }
\end{aligned}
$$

An oriented arc-coloring of an oriented graph $G$ is an oriented vertex-coloring of its line digraph $L D(G)$ (recall that $L D(G)$ is given by $V(L D(G))=A(G)$ and $a b \in$ $A(L D(G))$ if there exists $u, v, w \in V(G)$ such that $a=u v$ and $b=v w)$. We say that an oriented graph $G$ is $H$-arc-colorable if there exists a homomorphism $\varphi$ from $L D(G)$ to $H$. The mapping $\varphi$ is then an $H$-arc-coloring, or simply an arc-coloring, of $G$. Therefore, an oriented arc-coloring $\varphi$ of $G$ must satisfy $(i)$ $\varphi(u v) \neq \varphi(v w)$ whenever $u v$ and $v w$ are two consecutive arcs in $G$, and $(i i) \varphi(v w) \neq \varphi(x y)$ whenever $u v, v w, x y, y z \in A(G)$ with $\varphi(u v)=$ $\varphi(y z)$. Note that these two conditions ensure that any two arcs belonging to a directed path of length two or three must get distinct colors in any oriented arc-coloring. Also note that two arcs incoming to (resp. outgoing from) the same vertex can get the same color since the two corresponding vertices in $L D(G)$ are not adjacent and do not belong to a directed 2-path.

The oriented chromatic index of $G$, denoted by $\chi_{o}^{\prime}(G)$, is the smallest order of an oriented graph $H$ such that $G$ is $H$-arc-colorable. The oriented chromatic index $\chi_{o}^{\prime}(\mathcal{F})$ of a class of oriented graphs $\mathcal{F}$ is defined as the maximum of $\chi_{o}^{\prime}(G)$ taken over all graphs $G$ in $\mathcal{F}$.

Let $G$ and $H$ be two oriented graphs and $\varphi$ be a homomorphism from $G$ to $H$. It is not difficult to check that the mapping $\varphi^{\prime}$ : $A(G) \rightarrow V(H)$, given by $\varphi^{\prime}(x y)=\varphi(x)$ for every arc $x y$ in $G$ is a homomorphism from $L D(G)$ to $H$. Hence we have:

Observation 2 [7] For every oriented graph $G, \chi_{o}^{\prime}(G) \leq \chi_{o}(G)$.

From that, we get that every oriented outerplanar graph has oriented chromatic index at most 7 . Our second result gives a complete classification of the oriented chromatic indexes of oriented outerplanar graphs with given girth:

\section{Theorem 3}

1. $\chi_{o}^{\prime}\left(\mathcal{O}_{3}\right)=7$,

2. $\chi_{o}^{\prime}\left(\mathcal{O}_{4}\right)=6$,

3. $\chi_{o}^{\prime}\left(\mathcal{O}_{g}\right)=5$ for every $g, 5 \leq g \leq 9$,

4. $\chi_{o}^{\prime}\left(\mathcal{O}_{g}\right)=4$ for every $g, g \geq 10$. 
This paper is organised as follows. We give in Section 2 some notation and preliminary results that will be used later. We prove Theorem 1 in Section 3 and Theorem 3 in Section 4. Finally, we discuss the case of oriented partial 2-trees in Section 5.

\section{Notation and preliminary results}

In the rest of the paper, we will use the following notation. A $k$-vertex is a vertex of degree $k$. The minimum degree of a graph $G$ is denoted by $\delta(G)$. If $u v$ is an arc, $u$ is a predecessor of $v$ and $v$ is a successor of $u$. For a given vertex $u$ in $G$, we denote by $N_{G}^{+}(u)$ (resp. $\left.N_{G}^{-}(u)\right)$ the set of successors (resp. predecessors) of $u$ in $G$.

A $k$-path in $G$ is a sequence $x_{0} x_{1} \ldots x_{k}$ such that $x_{i} x_{i+1} \in A(G)$ or $x_{i+1} x_{i} \in A(G)$ for every $i, 0 \leq i<k$. A directed $k$-path in $G$ is a sequence $x_{0} x_{1} \ldots x_{k}$ such that $x_{i} x_{i+1} \in A(G)$ for every $i, 0 \leq i<k$. The notions of a $k$ cycle and of a directed $k$-cycle are defined in a similar way.

For a graph $G$ and a vertex $v$ of $V(G)$, we denote by $G \backslash v$ the graph obtained from $G$ by removing $v$ together with the set of its incident arcs. This notion is extended to sets of vertices in a standard way.

Let $G$ be an oriented graph and $f$ be an oriented arc-coloring of $G$. For a given vertex $v$ of $G$, we denote by $C_{f}^{+}(v)$ and $C_{f}^{-}(v)$ the outgoing color set of $v$ (i.e. the set of colors of the arcs outgoing from $v$ ) and the incoming color set of $v$ (i.e. the set of colors of the arcs incoming to $v$ ), respectively.

The upper bounds of Theorems 1 and 3 will be obtained by proving that the corresponding oriented outerplanar graphs are $T$-vertexor $T$-arc-colorable for some tournament $T$. The three tournaments that will be used are depicted in Figure 1.

The tournament $T_{4}$ is the only tournament on four vertices containing a directed 4-cycle.

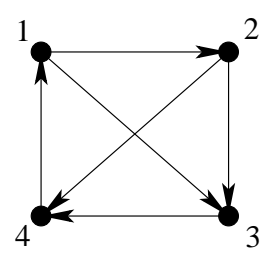

(a) The tournament $T_{4}$

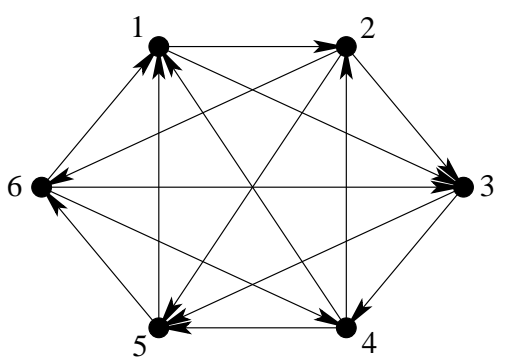

(c) The tournament $T_{6}$

Figure 1: Three target tournaments

Note that the tournament $T_{5}$ is a circular tournament and thus is arc-transitive. Moreover, this tournament has the following property:

Proposition 4 [2] For every pair of (not necessarily distinct) vertices $u, v \in V\left(T_{5}\right)$, there exists an oriented 4-path connecting $u$ with $v$ for any of the 16 possible orientations of such an oriented 4-path.

By a case analysis, it is tedious but not difficult to prove the following:

Proposition 5 For every pair of (not necessarily distinct) vertices $u, v \in V\left(T_{6}\right)$, there exists an oriented 3-path connecting $u$ with $v$ for any of the 8 possible orientations of such an oriented 3-path.

Finally, we will extensively use the following obvious property of outerplanar graphs with high girth: 
Proposition 6 Every outerplanar graph $G$ with girth at least $k$ and $\delta(G) \geq 2$ contains a face of length $l \geq k$ with at least $l-2$ consecutive 2-vertices.

\section{The oriented chromatic number of outerplanar graphs}

In this section we prove our Theorem 1.

Proof of Theorem 1(1). We first prove that $\chi_{o}\left(\mathcal{O}_{4}\right) \leq 6$. To show that, we prove that every triangle-free outerplanar graph has a homomorphism to the tournament $T_{6}$ depicted in Figure 1(c). Let $H$ be a minimal (with respect to inclusion as a subgraph) triangle-free outerplanar graph having no homomorphism to $T_{6}$. We show that $H$ contains neither a 1 -vertex nor two adjacent 2 -vertices.

1. Suppose that $H$ contains a 1-vertex $u$. Then, due to the minimality of $H$, the triangle-free outerplanar graph $H^{\prime}=H \backslash$ $u$ admits an oriented $T_{6}$-vertex-coloring $f$. Since every vertex of $T_{6}$ has at least two successors and at least two predecessors, $f$ can easily be extended to $H$.

2. Suppose now that $H$ contains two adjacent 2 -vertices $v$ and $w$. Then, due to the minimality of $H$, the triangle-free outerplanar graph $H^{\prime}=H \backslash\{v, w\}$ admits an oriented $T_{6}$-vertex-coloring $f$. By Proposition $5, f$ can be extended to $H$.

We thus get a contradiction thanks to Proposition 6.

In [4], Hosseini Dolama constructed an oriented triangle-free outerplanar graph $G$ with oriented chromatic number 6 (see Figure 2). For completion, we prove now that $\chi_{o}(G) \geq 6$.

Assume to the contrary that $f$ is a $T$ vertex-coloring of $G$ for some tournament $T$ on five vertices $(V(T)=\{1,2,3,4,5\})$ and let $f(u)=1, f(v)=2, f(w)=3, f(x)=4$ and

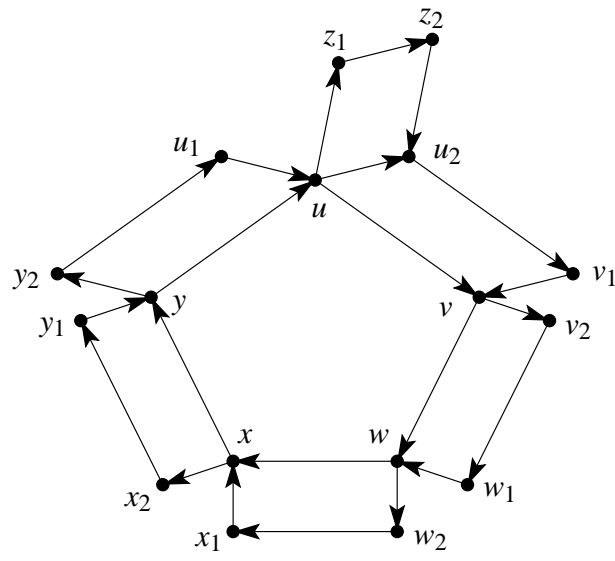

Figure 2: An oriented triangle-free outerplanar graph with oriented chromatic number 6

$f(y)=5$ (these five vertices belong to a directed 5-cycle in $G$ and thus must be assigned distinct colors).

Since every vertex in $\{u, v, w, x, y\}$ has 2 successors and 2 predecessors that are linked by a directed 2-path in $G$, each vertex of $T$ must have 2 predecessors and 2 successors. Hence, $T$ is necessarily the tournament $T_{5}$ depicted in Figure 1(b). (Note that since the tournament $T_{5}$ is arc-transitive, we may still assume w.l.o.g. that $f(u)=1, f(v)=2$, $f(w)=3, f(x)=4$ and $f(y)=5$.)

Finally, observe that we necessarily have $f\left(u_{2}\right)=3$ and that we cannot color the remaining vertices $z_{1}$ and $z_{2}$ since there is no directed 3-path in $T_{5}$ from 1 to 3 .

This completes the proof of Theorem 1(1).

Proof of Theorem 1(2). We first prove that $\chi_{o}\left(\mathcal{O}_{g}\right) \leq 5$ for every $g, g \geq 5$. Note that it is enough to consider the case $g=5$. More precisely, we prove that every outerplanar graph with girth at least 5 has a homomorphism to the tournament $T_{5}$ depicted in Figure 1(b).

Let $H$ be a minimal (with respect to inclusion as a subgraph) outerplanar graph with 
girth 5 having no homomorphism to $T_{5}$. We show that $H$ contains neither a 1-vertex nor three adjacent 2-vertices.

1. Suppose that $H$ contains a 1-vertex $u$. Then, due to the minimality of $H$, the outerplanar graph $H^{\prime}=H \backslash u$ (which has girth at least 5) admits an oriented $T_{5}$ vertex-coloring $f$. Since every vertex of $T_{5}$ has two successors and two predecessors, $f$ can easily be extended to $H$.

2. Suppose now that $H$ contains three adjacent 2-vertices $u, v$ and $w$. Then, due to the minimality of $H$, the outerplanar graph $H^{\prime}=H \backslash\{u, v, w\}$ (which has girth at least 5) admits an oriented $T_{5}$-vertexcoloring $f$. By Proposition $4, f$ can be extended to $H$.

We thus get a contradiction thanks to Proposition 6.

In [5], Nesetril et al. constructed for every $g, g \geq 3$, an oriented outerplanar graph $G_{g}$ with girth at least $g$ which has no homomorphism to the tournament $T_{4}$. We recall this construction here.

Let $P_{g}$ be the oriented path on $\left\lfloor\frac{g}{2}\right\rfloor$ vertices whose edges have alternatively forward and backward direction and let $u$ and $v$ denote its end-vertices. The graph $G_{g}$ is then constructed as follows : let $x_{1} x_{2} \ldots x_{p}$ be a directed cycle on $p \geq g$ vertices, such that $p \equiv 1$ or $2(\bmod 3)$. To every vertex $x_{i}$ attach two copies of $P_{g}$ by identifying the two $u$-vertices with $x_{i}$ and adding an arc (with any direction) linking the two $v$-vertices. The graph $G_{g}$ thus obtained has clearly girth $g$ or $g+1$. Moreover, for every homomorphism $f: G_{g} \rightarrow T_{4}$, one vertex $x_{i}$ at least satisfies $f\left(x_{i}\right)=3$. It is then easy to check that the two $v$-vertices of the paths attached to $x_{i}$ are mapped to the same vertex of $T_{4}$, namely 0 or 3 depending on the parity of $\left\lfloor\frac{g}{2}\right\rfloor$. Since these two vertices are joined by an arc in $G_{g}$ we obtain the desired contradiction and the result follows.

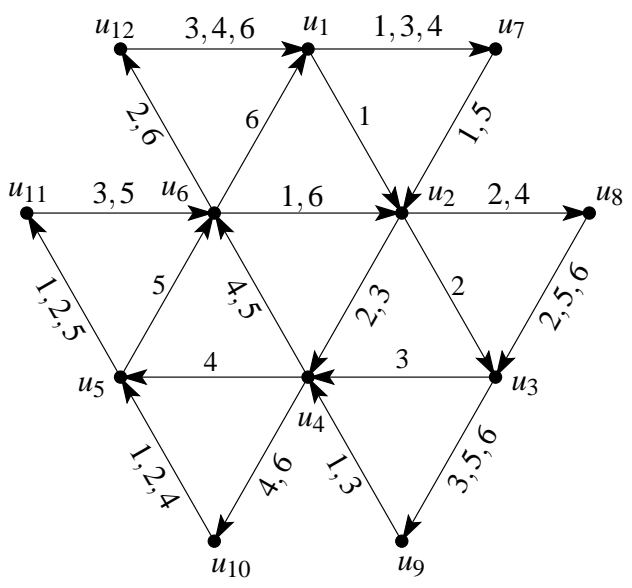

Figure 3: An oriented outerplanar graph with oriented chromatic index 7

This completes the proof of Theorem 1(2).

\section{The oriented chromatic in- dex of outerplanar graphs}

In this section we prove our Theorem 3.

Proof of Theorem 3(1). In [9], Sopena proved that every outerplanar graph has oriented chromatic number at most 7. By Observation 2 , we thus get that the oriented chromatic index of every outerplanar graph is at most 7 .

To show that this bound is tight, we shall prove that the oriented outerplanar graph $G$ depicted on Figure 3 has oriented chromatic index 7 .

Assume to the contrary that $f$ is an arccoloring of $G$ using at most six colors (say $1,2,3,4,5,6)$. Necessarily, the colors of the $\operatorname{arcs} u_{1} u_{2}, u_{2} u_{3}, u_{3} u_{4}, u_{4} u_{5}, u_{5} u_{6}$ and $u_{6} u_{1}$ are pairwise distinct since any two such arcs belongs to a directed 2- or 3-path in $G$. Suppose w.l.o.g. that $f\left(u_{i} u_{j}\right)=i$ (as drawn on Figure 3). This forbids for each remaining uncolored arc 3 or 4 colors. The sets of avail- 
able colors for each of these arcs are given in Figure 3.

Suppose first that $f\left(u_{6} u_{2}\right)=6$ and $f\left(u_{2} u_{4}\right)=3$. This implies $f\left(u_{11} u_{6}\right)=5$ and $f\left(u_{4} u_{10}\right)=4$ and then $f\left(u_{10} u_{5}\right)=1$ and $f\left(u_{5} u_{11}\right)=2$ since we already have $1 \rightarrow 2$ with $u_{1} u_{2}$ and $u_{2} u_{3}$. Therefore, since we have $2 \rightarrow 5$ with $u_{5} u_{11}$ and $u_{11} u_{6}$ (resp. $4 \rightarrow 1$ with $u_{4} u_{10}$ and $u_{10} u_{5}$ ), we necessarily have $f\left(u_{7} u_{2}\right)=1$ (resp. $\left.f\left(u_{2} u_{8}\right)=2\right)$. Finally, this implies $f\left(u_{1} u_{7}\right)=4$ and $f\left(u_{8} u_{3}\right)=5$ and there is no remaining available color for $u_{4} u_{6}$.

Suppose now that $f\left(u_{6} u_{2}\right)=1$ and $f\left(u_{4} u_{6}\right)=4$. This implies $f\left(u_{2} u_{8}\right)=2$ and $f\left(u_{9} u_{4}\right)=3$ and then $f\left(u_{8} u_{3}\right)=5$ and $f\left(u_{3} u_{9}\right)=6$ since we already have $5 \rightarrow 6$ with $u_{5} u_{6}$ and $u_{6} u_{1}$. Therefore, since we have $6 \rightarrow$ 3 with $u_{3} u_{9}$ and $u_{9} u_{4}$ (resp. $2 \rightarrow 5$ with $u_{2} u_{8}$ and $\left.u_{8} u_{3}\right)$, we necessarily have $f\left(u_{4} u_{10}\right)=4$ (resp. $f\left(u_{6} u_{12}\right)=6$ ). Finally, this implies $f\left(u_{10} u_{5}\right)=2$ and $f\left(u_{12} u_{1}\right)=3$ and there is no remaining available color for $u_{2} u_{4}$.

Finally, suppose that $f\left(u_{2} u_{4}\right)=2$ and $f\left(u_{4} u_{6}\right)=5$. This implies $f\left(u_{7} u_{2}\right)=1$ and $f\left(u_{6} u_{12}\right)=6$ and then $f\left(u_{12} u_{1}\right)=3$ and $f\left(u_{1} u_{7}\right)=4$ since we already have $3 \rightarrow 4$ with $u_{3} u_{4}$ and $u_{4} u_{5}$. Therefore, since we have $6 \rightarrow 3$ with $u_{6} u_{12}$ and $u_{12} u_{1}$ (resp. $4 \rightarrow 1$ with $u_{1} u_{7}$ and $\left.u_{7} u_{2}\right)$, we necessarily have $f\left(u_{11} u_{6}\right)=5$ (resp. $f\left(u_{9} u_{4}\right)=3$ ). Finally, this implies $f\left(u_{5} u_{11}\right)=1$ and $f\left(u_{3} u_{9}\right)=6$ and there is no remaining available color for $u_{6} u_{2}$.

Therefore, the colors of $u_{2} u_{4}, u_{4} u_{6}$ and $u_{6} u_{2}$ are respectively either $3,5,1$ or $2,4,6$.

Suppose first that $f\left(u_{2} u_{4}\right)=3, f\left(u_{4} u_{6}\right)=$ 5 and $f\left(u_{6} u_{2}\right)=1$. This implies $f\left(u_{9} u_{4}\right)=3$ and $f\left(u_{11} u_{6}\right)=5$. Then, we necessarily have $f\left(u_{3} u_{9}\right)=6$ and $f\left(u_{5} u_{11}\right)=2$. Finally, this implies $f\left(u_{4} u_{10}\right)=4$, and there is no remaining available color for $u_{10} u_{5}$.

Finally, suppose that $f\left(u_{2} u_{4}\right)=2$, $f\left(u_{4} u_{6}\right)=4$ and $f\left(u_{6} u_{2}\right)=6$. This implies $f\left(u_{4} u_{10}\right)=4$ and $f\left(u_{2} u_{8}\right)=2$. Then, we necessarily have $f\left(u_{10} u_{5}\right)=1$ and $f\left(u_{8} u_{3}\right)=5$. Finally, this implies $f\left(u_{9} u_{4}\right)=3$, and there is no remaining available color for $u_{3} u_{9}$.
Hence, there exists no tournament $T$ on six vertices such that $G$ is $T$-arc-colorable, which completes the proof of Theorem 3(1).

Proof of Theorem 3(2). By Theorem 1(1), every triangle-free outerplanar has oriented chromatic number at most 6 and thus, by Observation 2 , has oriented chromatic index at most 6 .

To show that this bound is tight, we shall prove that the oriented triangle-free outerplanar graph $G$ depicted on Figure 4(a) has oriented chromatic index 6 .

Assume to the contrary that $f$ is an oriented arc-coloring of $G$ using at most 5 colors, say $1,2,3,4,5$. Necessarily, the colors of the arcs $u v, v w, w x, x y$ and $y u$ have to be pairwise distinct. Suppose w.l.o.g. that $f(u v)=1, f(v w)=2, f(w x)=3, f(x y)=4$ and $f(y u)=5$.

We first prove that $f$ is an oriented $T_{5}$-arccoloring ( $T_{5}$ is the tournament depicted on Figure 1(b)). Suppose to the contrary that $f$ is an oriented $T_{5}^{\prime}$-arc-coloring of $G$, with $T_{5}^{\prime} \neq$ $T_{5}$. We may assume w.l.o.g. that $N_{T_{5}^{\prime}}^{+}(1)=$ $\{2\}$, which implies $N_{T_{5}^{\prime}}^{-}(1)=\{3,4,5\}$. This successively implies $f\left(v v_{2}\right)=2, f\left(w_{1} w\right)=$ $5, f\left(w w_{2}\right)=3, f\left(x_{1} x\right)=2, f\left(x x_{2}\right)=4$, $f\left(y_{1} y\right)=2, f\left(v_{1} v\right)=1$ and $f\left(u u_{2}\right)=3$. Then, the only avalaible color for $u_{1} u$ and $y y_{2}$ is 5 , a contradiction.

Therefore, $f$ is an oriented $T_{5}$-arc-coloring of $G$. (Note that since the tournament $T_{5}$ is arc-transitive, we may still assume w.l.o.g. that $f(u v)=1, f(v w)=2, f(w x)=3$, $f(x y)=4$ and $f(y u)=5$.

Observe now that we have either $f\left(u u_{2}\right)=$ 1 or $f\left(u u_{2}\right)=2$. In each case, the color of $f\left(u u_{2}\right)$ fixes the colors of all but five $\operatorname{arcs}$ of $G$ (see Figure 4(b) for the case $f\left(u u_{2}\right)=1$ and Figure 4(c) for the case $f\left(u u_{2}\right)=2$ ).

To complete the $T_{5}$-arc-coloring $f$, we thus need a directed 3 -path in $T_{5}$ from color 1 to 3 (when $f\left(u u_{2}\right)=1$ ) or from color 3 to 5 (when $f\left(u u_{2}\right)=2$ ). Since such paths do not exist in $T_{5}$, we get a contradiction 


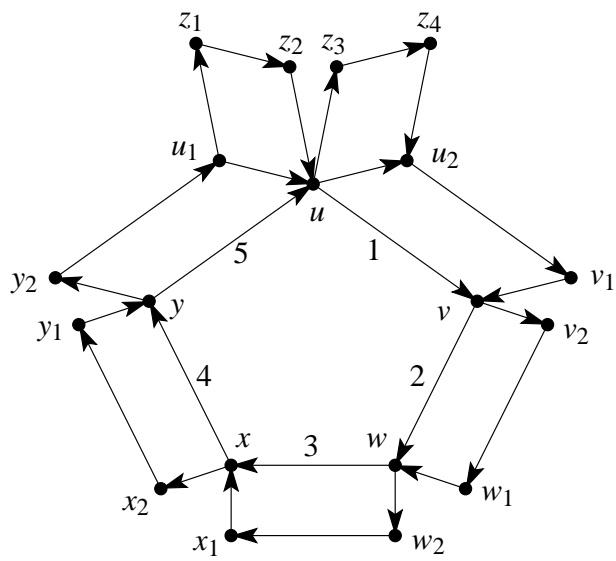

(a)

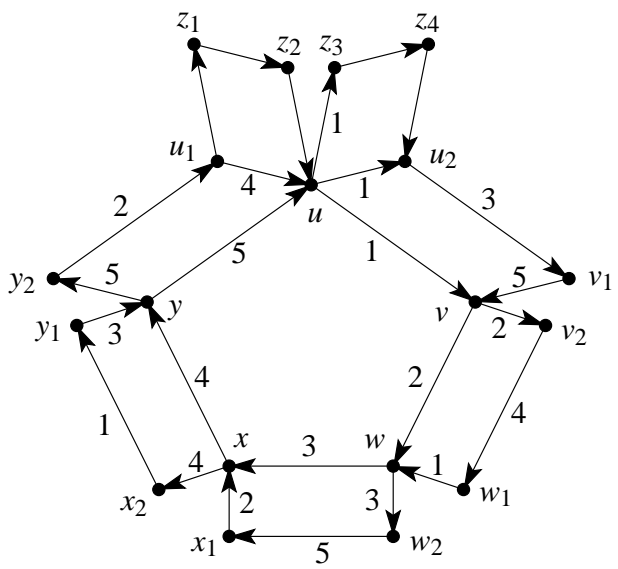

(b)

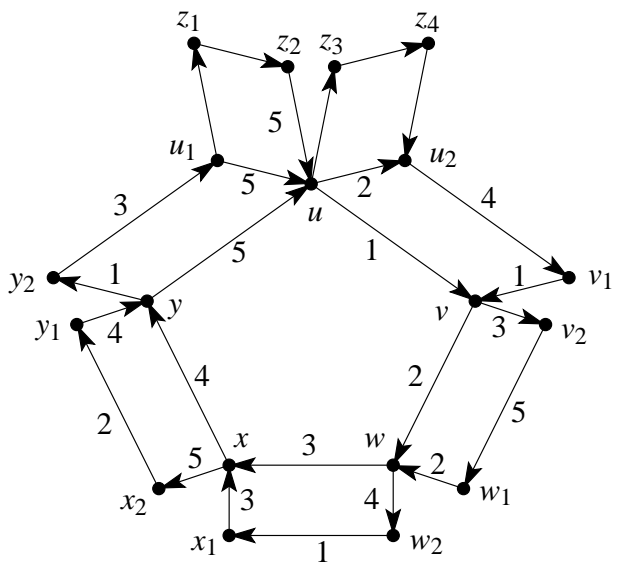

(c)

Figure 4: An oriented triangle-free outerplanar graph with oriented chromatic index 6

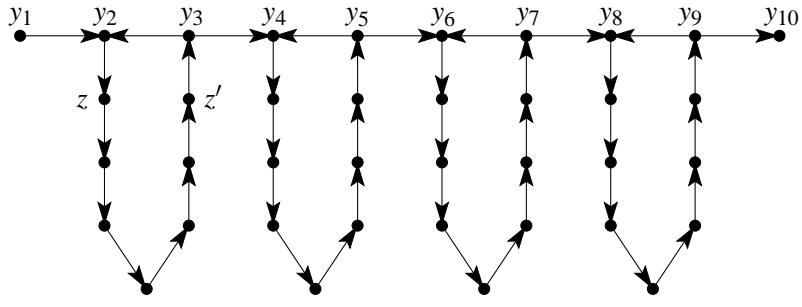

Figure 5: The configuration $H$ for the proof of Theorem 3(3)

which completes the proof of Theorem 1(1).

Proof of Theorem 3(3). By Theorem 1(2), every outerplanar graph with girth $g, 5 \leq g \leq$ 9 , has oriented chromatic number at most 5 and thus, by Observation 2, has oriented chromatic index at most 5 .

To show that this bound is tight, we construct an outerplanar graph with girth 9 and oriented chromatic index 5 .

Consider the configuration $H$, made of an alternating path of ten vertices and four directed 9-cycles arranged as depicted on Figure 5. Observe first that the tournament $T_{4}$ (depicted on Figure 1(a)) contains only two directed 3-cycles, namely 124 and 134. Hence, any oriented $T_{4}$-arc-coloring of the directed 9-cycle has the form $1 x 41 x 41 x 4$ with $x \in\{2,3\}$. Let now $h$ be a $T_{4}$-arc-coloring of $H$ with $h\left(y_{1} y_{2}\right)=1$. We then necessarily have $h\left(y_{2} z\right) \in\{2,3\}$ and, by the observation before, $h\left(z^{\prime} y_{3}\right)=4$, which implies $h\left(y_{3} y_{2}\right)=h\left(y_{3} y_{4}\right)=1$. By repeating this argument for the four directed 9-cycles of $H$, we get $h\left(y_{9} y_{10}\right)=1$.

Consider finally the oriented graph $G$ obtained from the directed 10-cycle $C_{10}=$ $x_{0} x_{1} \ldots x_{9}$ by gluing ten copies of $H$ to the ten arcs of $C_{10}$ by identifying $y_{1}$ with $x_{i}$ and $y_{10}$ with $x_{i-1}$ (subscripts are taken modulo 10) for every $i, 0 \leq i \leq 9$. The so-obtained graph $G$ has clearly girth 9 . If $T$ is a tournament on four vertices such that $G$ admits a $T$-arc-coloring then $T$ must contain a directed 4-cycle since $G$ contains a directed 10-cycle. 
Therefore, $T$ is necessarily the tournament $T_{4}$.

Let now $f$ be a $T_{4}$-arc-coloring of $G$. Since $10 \equiv 1(\bmod 3)$, the color 4 must be used on some arc of the cycle $C_{10}$, say $f\left(x_{0} x_{1}\right)=4$. Considering the copy of $H$ glued to $x_{0} x_{1}$, we necessarily have $f\left(x_{1} y_{2}\right)=1$ and thus $f\left(y_{9} x_{0}\right)=1$ from the above discussion, a contradiction.

Hence, the graph $G$ has no $T_{4}$-arc-coloring and has thus oriented chromatic index 5 . That completes the proof of Theorem 1(2).

In order to prove Theorem 3(4) we need the following technical results.

We say that a $T_{4}$-arc-coloring $f$ of an oriented graph $G$ is good if

1. $\forall u \in V(G), C_{f}^{+}(u) \in\{\{1\},\{2\},\{3\},\{4\}$, $\{2,3\},\{3,4\}\}$,

2. $\forall u \in V(G), C_{f}^{-}(u) \in\{\{1\},\{2\},\{3\},\{4\}$, $\{1,2\},\{2,3\}\}$.

Ochem et al. [7] proved the following:

Proposition 7 [7] Let $P=v_{0}, v_{1}, \ldots, v_{9}, v_{10}$ be an oriented 10-path. Any good $T_{4}$-arccoloring of $P^{\prime}=P \backslash\left\{v_{2}, \ldots, v_{8}\right\}$ can be extended to a good $T_{4}$-arc-coloring of $P$.

A computer check shows the following:

Proposition 8 Let $P=u, v_{1}, v_{2}, \ldots, v_{8}, w$ be an oriented 9-path. Any good $T_{4}$-arccoloring of $P^{\prime}=P \backslash\left\{v_{2}, \ldots, v_{7}\right\}$ such that $C_{f}^{-}(u) \cap C_{f}^{+}(w) \neq \emptyset$ or $C_{f}^{+}(u) \cap C_{f}^{-}(w) \neq \emptyset$ can be extended to a good $T_{4}$-arc-coloring of $P$.

We are now able to prove Theorem 3(4).

Proof of Theorem 3(4). We first prove that $\chi_{o}^{\prime}\left(\mathcal{O}_{g}\right) \leq 4$ for every $g, g \geq 10$. Note that it is enough to consider the case $g=10$. More precisely, we prove that every outerplanar graph with girth at least 10 has a homomorphism to the tournament $T_{4}$ depicted in Figure 1(a).
Let $H$ be a minimal (with respect to inclusion as a subgraph) outerplanar graph with girth 10 having no homomorphism to $T_{4}$. We show that $H$ contains neither a 1-vertex nor a face $F$ of length $l \geq 10$ with $l-2$ consecutive 2 -vertices.

1. Suppose that $H$ contains a 1-vertex $u$. Let $v$ be its neighbor and suppose that $u v \in A(H)$. The graph $H^{\prime}=H \backslash u$ is an outerplanar graph with girth at least 10 and, due to minimality of $H$, admits a good $T_{4}$-arccoloring $f$. Therefore, we have $C_{f}^{+}(v) \in$ $\{\{1\},\{2\},\{3\},\{4\},\{2,3\},\{3,4\}\}$. For each possible case, there clearly exists a predecessor in $T_{4}$ that can be used to extend $f$ to a good $T_{4}$-arc-coloring of $H$. The proof of the case $v u \in A(H)$ is similar.

2. Suppose now that $H$ contains a face $F$ of length $l \geq 10$ with $l-2$ consecutive 2 -vertices. We consider two cases:

(a) $l=10$.

Let $F=u, v_{1}, v_{2}, \ldots, v_{8}, w$. Due to the minimality of $H$, the graph $H^{\prime}=H \backslash\left\{v_{2}, \ldots, v_{7}\right\}$ admits a good oriented $T_{4}$-arc-coloring $f$. Moreover, since $u w \in A(H)$ or $w u \in$ $A(H)$, we have $C_{f}^{-}(u) \cap C_{f}^{+}(w) \neq \emptyset$ or $C_{f}^{+}(u) \cap C_{f}^{-}(w) \neq \emptyset$ respectively. By Proposition $8, f$ can be extended to $H$.

(b) $l>10$.

The face $F$ contains nine consecutive 2 -vertices, say $u_{1}, \ldots, u_{9}$. Due to the minimality of $H$, the graph $H^{\prime}=H \backslash\left\{u_{2}, \ldots, u_{8}\right\}$ admits a good $T_{4}$-arc-coloring $f$. By Proposition 7 , $f$ can be extended to $H$.

We thus get a contradiction thanks to Proposition 6.

We finally prove that for every $k \geq 3$ there exist outerplanar graphs with girth $k$ and ori- 
ented chromatic index at least 4, using a construction proposed in [7]. Observe first that any arc-coloring of a directed cycle of length $p, p \equiv 1$ or $2(\bmod 3)$, must use at least 4 colors. Hence, the graph $G_{k}$ made of two directed cycles sharing one arc, one with length $k$, the other with length $p, p \equiv 1$ or $2(\bmod 3)$, $p \geq k$, has the desired property.

This completes the proof of Theorem 3(4).

\section{Oriented partial 2-trees}

Recall that a partial 2 -tree is a $K_{4}$-minorfree graph (also knwon in the litterature as a series-parallel graph) and that the class of oriented partial 2-trees strictly contains the class of outerplanar graphs.

A natural question is thus to extend our results to the case of partial 2-trees.

As discussed before, Sopena proved in [9] that every oriented partial 2-tree has oriented chromatic number at most 7 and that this bound is tight. Moreover, we can prove that this bound is also tight for oriented trianglefree partial 2-trees:

Theorem 9 The exist oriented triangle-free partial 2-trees with oriented chromatic number 7 .

Proof. We shall prove that the oriented triangle-free partial 2-tree $G$ depicted on Figure 6 has oriented chromatic number 7 .

Suppose that $G$ admits an oriented 6vertex-coloring $f$. Clearly, the colors of the vertices $u, w_{1}, w_{2}, w_{3}, w_{4}, v$ have to be pairwise distinct. W.l.o.g., we assume that $f(u)=$ $1, f(v)=2, f\left(w_{1}\right)=3, f\left(w_{3}\right)=4, f\left(w_{3}\right)=5$ and $f\left(w_{4}\right)=6$.

Suppose first that $f(w)=3$ (resp. $f(w)=$ 4). Then, the two vertices $v_{2}$ and $v_{4}$ (resp. $v_{1}$ and $v_{3}$ ) must get distinct colors and the two only available colors are 2 and 5 (resp. 2 and 6). Therefore, we will have an arc in $G$ from the color 2 to the color 1 (resp. from 1

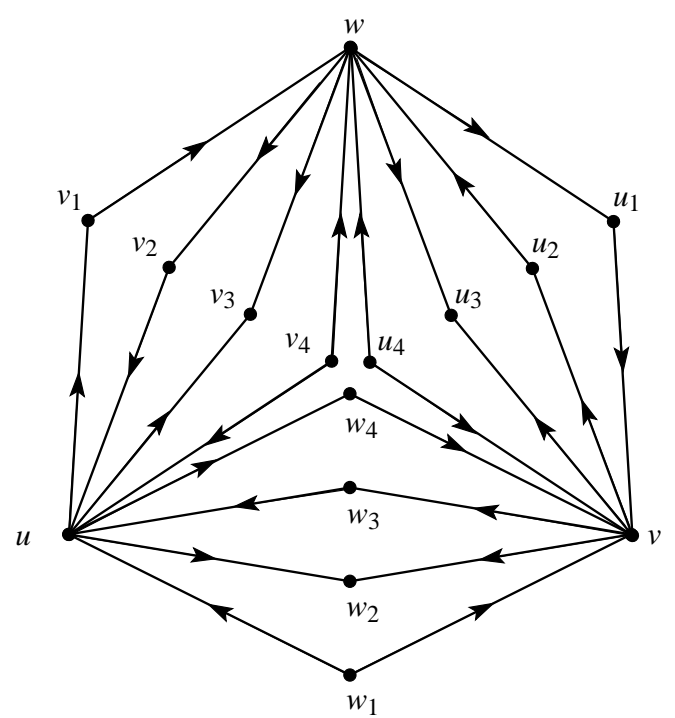

Figure 6: An oriented triangle-free partial 2tree with oriented chromatic number 7

to 2 ). Now, the vertices $u_{1}$ and $u_{4}$ (resp. $u_{2}$ and $u_{3}$ ) must get distinct colors and the only two available colors are 1 and 6 (resp. 1 and $5)$. We get a contradiction since we will then have an arc in $G$ from the color 1 to the color 2 (resp. from 2 to 1 ).

The remaining cases $f(w)=5$ and $f(w)=6$ lead to a contradiction in a similar way.

Finally note that Theorem 3(1) implies that the oriented chromatic index of every oriented partial 2-tree is at most 7 and that this bound is tight.

It would thus be interesting to determine the exact value of the oriented chromatic number (resp. of the oriented chromatic index) of oriented partial 2-trees with girth $k$, for every $k \geq 5$ (resp. $k \geq 4$ ).

\section{References}

[1] O. V. Borodin and A. O. Ivanova. An oriented 7-colouring of planar graphs with girth at least 7. Siberian Electronic Mathematical Reports, 2:222-229, 2005. 
[2] O. V. Borodin, A. V. Kostochka, J. Nešetřil, A. Raspaud, and E. Sopena. On the maximum average degree and the oriented chromatic number of a graph. Discrete Math., 206:77-89, 1999.

[3] B. Courcelle. The monadic second orderlogic of graphs VI : on several representations of graphs by relationnal stuctures. Discrete Appl. Math., 54:117-149, 1994.

[4] M. Hosseini Dolama. Contribution à l'étude de quelques problèmes de coloration de graphes (in french). PhD thesis, LaBRI, Université Bordeaux I, 2005.

[5] J. Nešetřil, A. Raspaud, and E. Sopena. Colorings and girth of oriented planar graphs. Discrete Math., 165-166:519-530, 1997.

[6] P. Ochem. Oriented colorings of triangle-free planar graphs. Inform. Process. Lett., 92:7176, 2004.

[7] P. Ochem, A. Pinlou, and E. Sopena. On the oriented chromatic index of oriented graphs. Research Report RR-1390-06, LaBRI, Université Bordeaux 1, 2006.

[8] A. Raspaud and E. Sopena. Good and semistrong colorings of oriented planar graphs. Inform. Process. Lett., 51(4):171-174, 1994.

[9] E. Sopena. The chromatic number of oriented graphs. J. Graph Theory, 25:191-205, 1997.

[10] E. Sopena. Oriented graph coloring. Discrete Math., 229(1-3):359-369, 2001.

[11] D. R. Wood. Acyclic, star and oriented colourings of graph subdivisions. Discrete Math. and Theoret. Comput. Sci., 7(1):3750, 2005. 